Das vierte Geschwür ist erbsengroß und findet sich handbreit oberhalb der Ellenbeuge über dem medialen Teil des Bizeps.

Alle diese Ulzera sind wie mit einem Locheisen scharf ausgestanzt, von gummaähnlichem Aussehen, auf der Unterlage gut verschieblicli und gehen $3-5 \mathrm{~mm}$ in die Tiefe. Sie sind auffallend indolent und sondern in mäßiger Menge ein schleimiges, fadenziehendes Sekret ab. Der Gesclıwürsgrund zeigt teils frischrote Granulationen, teils wenig schleimigen Belag. Die Umgebung der Ulzera ist wenig infiltriert und von hellrotvioletter Verfärbung, die nach außen diffus in normale Hantfarbe übergeht. Die linke Kubitaldrüse ist tastbar, sonst keine Lymphadenitis. Die Röntgenaufinahme der linken Hand ergab: ,halb-

Aus der Hautabteilung des Städtischen Krankenhauses Karlsruhe. (Gen.-Oberarzt a. D. Dr. v. Pezold.)

\title{
Ein Fall von Sporotrichose.
}

\section{Von Dr. A. Geiger.}

Die Sporotrichose gehört zu den erst kürzlich bekannt gewordenen Hauterkrankungen. Ihr Vorkommen ist, wie uns ein Blick in die Lehrbiitcler zeigt, in Deutschland selten; häufiger wird sie in Frankreich beobachtet. Französischen Autoren, besonders de Beurmann und Gougerot, verdanken wir ihre eingehende Erforschung und genaue Darstellung.

Verursacht wird die Sporotrichose durch Fadeupilze, Sporotricha, am häufigsten durch das Sporotrichon Beurmanni. Ihre Eintrittspforte in den menschlichen Körper bilden teils bestehende Wunden, teils sind sie unbekannt, und es wird der Verdauungstraktus als solche vermutet. Die verursaclıten Hantverändernugen sind äıßerst mannigfach. Besonders charakteristisch für die Sporotrichose ist die Billung von indolenten Knoten, die erweichen, sich in indolente gummöse Ulzera umwandeln und durch derbe Lymphstränge untereinandel verbunden sind. Das Krankheitsbild erinnert in mancherlei Beziehung namentlich an Lues und Tuberkulose.

Ott köl111en wir die Eintrittspforte der Sporotrichose als „sporotrichotischen Schanker" nachweisen. In solchen Fällen pflegt die Erkrankung auf das zugehörige Lymphgebiet beschränkt zu bleiben, doch sind anch disseminierte Fälle bekannt geworden.

Ein Fall von Sporotrichose, der sich durch seinen typischen Verlauf auszeichnet, $\mathrm{kam}$ in der letzten Zeit hier zur Beobachtung. Bei der Seltenheit dieser Dermatomykose dürfte dessen Mitteilung von lnteresse sein.

Der jetzt 18jährige Patient erlitt im Septenber 1918 in einem böhnischen Bergwerke eine Quctsclıung des linken Handrückens. Die beiden Quetschwunden verheilten innerhalb 6 Wochen unter essigsauren Tonerdeverbänden1 und Moorsalzbädlern. 3-4 Wochen 11ach den1 Abheilen brachen beide Narben wieder auf. Etwa 6--8 Wochen später, im Januar 1919, bildeten sich proximalwärts von diesen Wunden auf dem linken Handrücken 2 Knoten, ,die sich wie Drüsen anfühlten". Diese erweichten langsam und brachen auf, wodurch 2 scharf ausgestanzte Geschwïre entstanden. Bis zum Januar 1921 fand, abgesehen von März bis Juni 1919, keine ärztliche Belıandling statt. Eine Veränderung der 4 Geschwüre trat in dieser Zeit, während welcher Patient teils als tschechischer Soldat, teils als Bergun1 Fabrikarbeiter ungestört Dienst tat, nicht ein.

Im Januar 1921 kam der Patient ins Städtische Krankenhaus Karlsruhe, wo die Geschwüre mit feuchten Verbänden und Röntgen behandelt wurden. Ende Februar 1921 wurde Patient wegen anderweitiger Erkrankıng verlegt. Dadurch verloren wir ihn zunächst aus dem Auge. Erst Mitte April 1921 sahell wir den Patienten wieder. Inzwischeri liatten sich, Anfang März 1921, am linken Ober- und Unterarm 4 Knoten gebildet, die langsanı erweichten und inzidiert worden waren. Die Geschwüre auf dem Handrücken waren im März 11nd April 1921 unter Fortsetzung des Röntgens abgeheilt.

Der Befund war Mitte April 1021 folgender:

Gesund aussehender, kräftiger Patient. Die linke Hand ist magerer als die rechte. Auf dem linken Handrücken sehen wir 4 Narben, zwei über dem distalen Drittel der Metakarpen des Mittel- und Zeige. fingers, eine über dem proximalen Drittel der Mittelhandknochen des Ring- und Kleinfingers und eine über der Mitte des Metakarpus des Zeigefingers. Die zwei ersten Narben sind die der Quetschwunden. Sie sind etwa fünfzigpfennigstückgroß, nach der Mitte zu eingezogen, gering gefältelt, teilweise von wenig Schorf bedeckt und beide in ilırem Zentrum gering adlärent. Die zwei letzten Narben stammen von den inı Janılar 1919 entstandenen und später ulzerierten Knoten. Sie sind ungefähr pfenniggroß, gering verdickt, auf der Unterlage gut verschieblich, reaktionslos und ihr Rand gering wallartig aufgeworfen.

Anı linken Ober- bzw. Unterarm finden sich 4 Geschwüre. Das am Unterarm ist an der Radialseite, zweiquerfingerbreit unterhalb der Ellenbeuge, längsverlaufend, $11 / 2 \mathrm{~cm}$ breit und $21 / 2 \mathrm{~cm}$ lang, mit etwas aufgeworfenen Rändern. An der Radialseite des Oberarmes erblicken wir zweiquerfingerbreit oberhalb der Ellenbeuge ein von unten nach oben verlaufendes, $3 \mathrm{~cm}$ langes und $1 \frac{11 / 2}{\mathrm{~cm}}$ breites Ulkus. $1 \mathrm{~cm}$ unterhalb davon, direkt oberhalb des Gelenkes, ein linsengroßes Geschwür.

Diese 3 Geschwiire sind durch zwei bleistiftdicke, derbe, indolente Lymphstränge, über denen die Halit diffus blanrot verfärbt ist, untereiliander verbunden. mondförmiger Defekt mit Sequestereinlagerung am medialen Teil des
Metakarpus II unterhalb des distalen Köpfchens" (Quetschung!). Oberund Unterarm keine Knochenveränderung.

Hervorgehoben sei noch, daß Patient anch während der Beobachtung nie Beschwerden von seiten der Knoten bzw. Geschwüre hatte 11nd daß das Allgemeinbefinden dadurch keine Beeinträchtigung erfulır

Bei diesem Befunde mußte differentialdiagnostisch besonders an Lues und Tuberkulose gedacht werden. Tertiäre Lues, Gunnna, komnten wir durch den ständig negativen Wassernann, durch das Fehlen von anamuestischen und klinischen Auhaltspunkten sowie durch den ganzen Verlauf der Erkrankung ausschließen. Die Differentialdiagnose gegenüber Tuberkulose (Skrophuloderma, Bazin usw.) geht leicht aus dem Befunde hervor; näheres Eingehen daranf kan11 ich mir im Rahmen dieser Mitteilung ersparen.

Das ganze Bild ist das einer L. y m phang it is sporotrichotica g $11 \mathrm{mmos}$. Als Eintrittspforte der Erreger sehen wir die 2 QuetschWunden, von denell alls die regionäre lynıhogenle Ausbreitung des Krankheitsvirus erfolgte, was zur Bildung der Knoten und infolge deren Erweichung zu den beschriebenen Ulzera fülırte.

Der kulturelle Nachweis der irankheitserreger war uns nicht nögliclı. Die Sporotricha lassen sich zwar aus den geschlossenen Abszessen leicht im Kulturverfahren nachweisen. Aber geschlossenc Abszesse kamen uns leider nicht zu Gesicht, da sie anderwärts inzidiert worden waren. Die mit den Sekret der Ulzera geimpften Ratten ind Agarplatten ergaben, wie auch zu erwarten war, kein Ergebnis. Die Sporoagglutination kointen wir aus äußeren Gründen nicht anwenden.

Die Ulzera der Hand waren, wie gesagt, nach 2-3monatiger Röntgenbestrahlung und unter lokalen feuchten Verbänden abgeheilt Bei den Ulzera des Ober- und Unterarmes wandten wir nach Wiederïbernahme des Patienten die von Be $11 \mathrm{rm}$ an 11 angegebene Therapie, lokal Umschläge mit Jod-Jodkalilösung und intern 3--4 g Jodkal pro die, an. Auf diese Therapie hin setzte alsbald die Riickbildung der Erscheinungen, Reinigung der Wunden, Kräftign1119 der Granılatione11 und Verkleinerung ein.

Zum Schlusse möchte ich erwähnen, daß die Diagnose nachträglich durch Prof. Bettma n n (Heidelberg) und Prof. Rost (Freiburg) bestätigt wurde. 